\title{
Prevalência de depressão e outras doenças psiquiátricas em pacientes com histórico de acidente vascular encefálico
}

\author{
Prevalence of depression and other psychiatric diseases in patients with history of brain vascular
}

disease

Prevalencia de depresión y otras enfermedades psiquiátricas em pacientes con antecedentes de accidente vascular cerebral

\author{
Júlia Mello Lucena \\ ORCID: https://orcid.org/0000-0002-7545-9970 \\ Centro Universitário da Fundação Assis Gurgacz, Brasil \\ E-mail: juliamlucena@hotmail.com \\ Odirlei Antônio Magnagnagno \\ ORCID: https://orcid.org/0000-0003-0500-2251 \\ Centro Universitário da Fundação Assis Gurgacz, Brasil \\ E-mail: odirlei@fag.edu.br \\ Andrea Maria Rigo Lise \\ ORCID: https://orcid.org/0000-0003-1770-5288 \\ Centro Universitário da Fundação Assis Gurgacz, Brasil \\ E-mail: andrealise2094@hotmail.com
}

\begin{abstract}
Resumo
O acidente vascular encefálico (AVE) é definido como uma patologia neurológica que abrange três principais subtipos: hemorragia subaracnóidea, hemorragia intracerebral e isquemia cerebral. Comparando dados acerca do evento isquêmico em relação ao hemorrágico, há maior incidência de Acidente Vascular Encefálico Isquêmico (AVEi), que origina cerca de $85 \%$ dos casos. Dessa forma, este trabalho retrospectivo, quantitativo e observacional buscou descrever a prevalência de doenças psiquiátricas, classe de medicações utilizadas e associação entre sexo e idade com doenças em pacientes com histórico de AVE em atendimento ambulatorial entre 2015 e 2019. Os testes estatísticos utilizados foram Fisher e Qui-Quadrado, com nível de significância $\alpha=0,05$. A prevalência de depressão foi de $53 \%$ e de transtorno de ansiedade de $16 \%$ neste estudo. Houve associação de maior prevalência de demência vascular e epilepsia $(\mathrm{p}=0,05)$ nos pacientes do sexo masculino. Portanto, patologias psiquiátricas como depressão e ansiedade apresentam elevada prevalência em pacientes com histórico de AVE, sendo necessário acompanhamento multiprofissional.
\end{abstract}

Palavras-chave: Depressão; Acidente vascular encefálico; Transtorno de pânico; Demência vascular.

\begin{abstract}
Stroke is defined as a neurological pathology with three main subtypes: subarachnoid hemorrhage, intracerebral hemorrhage and cerebral ischemia. Comparing the ischemic event in relation to the hemorrhagic, there is greater incidence of Ischemic Stroke, which originates about $85 \%$ of the cases. Thus, this retrospective observational study sought to describe the prevalence of psychiatric illnesses, the used medication class and association between sex and age with illnesses in patients with a history of stroke with outpatient care between 2015 and 2019. The statistical tests used were Fisher and Chi-squared, with significance level $\alpha=0,05$. The prevalence of depression was $53 \%$ and anxiety disorder $16 \%$ in this study. There was an association of higher prevalence of vascular dementia and epilepsy ( $\mathrm{p}=0,05)$ in male patients. Therefore, psychiatric pathologies such as depression and anxiety have a high prevalence in patients with a history of stroke, requiring multiprofessional monitoring.
\end{abstract}

Keywords: Depression; Stroke; Panic disorder; Vascular dementia.

\section{Resumen}

El accidente cerebrovascular se define como una patología neurológica que abarca tres subtipos principales: hemorragia subaracnoidea, hemorragia intracerebral e isquemia cerebral. Comparando los datos sobre el evento isquémico en relación al evento hemorrágico, existe una mayor incidencia de Accidente Cerebrovascular Isquémico (ACV), que origina alrededor del $85 \%$ de los casos. Así, este trabajo retrospectivo, cuantitativo y observacional buscó describir la prevalencia de enfermedades psiquiátricas, clase de medicamentos utilizados y asociación entre sexo y edad con enfermedades en pacientes con antecedentes de accidente cerebrovascular atendidos en atención ambulatoria entre 2015 y 2019. Las pruebas estadísticas utilizadas fueron Fisher y Chi-Cuadrado, con nivel de significancia $\alpha=0,05$. La prevalencia de depresión fue del 53\% y el trastorno de ansiedad fue del 16\% en este estudio. Hubo una asociación de 
mayor prevalencia de demencia vascular y epilepsia $(\mathrm{p}=0,05)$ en pacientes masculino. Por tanto, patologías psiquiátricas como la depresión y la ansiedad tienen una alta prevalencia en pacientes con antecedentes de accidente cerebrovascular, requiriendo un seguimiento multiprofesional.

Palabras clave: Depresión; Infarto cerebral; Trastorno de pánico; Demencia vascular.

\section{Introdução}

O acidente vascular encefálico (AVE) é definido como uma patologia neurológica que abrange três principais subtipos: hemorragia subaracnóidea, hemorragia intracerebral e isquemia cerebral. Esta última pode ser decorrente de trombose, embolia ou hipoperfusão cerebral. As doenças que causam isquemia cerebral, na atualidade, são consideradas grandes problemas de saúde pública no mundo pela incidência e mortalidade crescentes. O AVE ocorre quando o distúrbio de circulação sanguínea cerebral - que pode causar o subtipo isquêmico ou hemorrágico - é iniciada por um déficit agudo e perdura por 24 horas ou mais, comprometendo a função cerebral rapidamente (Chaves et al, 2008; Hankey, 2016).

Comparando dados acerca do evento isquêmico em relação ao hemorrágico, há maior incidência de Acidente Vascular Encefálico Isquêmico (AVEi), que origina cerca de 85\% dos casos, sobre o Acidente Vascular Encefálico Hemorrágico (AVEh), que é responsável por aproximadamente 15\% (Ministério da Saúde, 2019).

Os fatores de risco envolvidos nessa patologia são variados e compreendem história familiar de eventos cerebrovascular, hipertensão arterial sistêmica (HAS), diabetes mellitus (DM), hiperlipidemia, etilismo, tabagismo, obesidade, sedentarismo, raça negra e idade avançada (Biller et al, 2004; Sarikaya et al, 2015; Caprio e Sorond, 2018).

Considera-se que, em muitos casos, a prevenção do AVE é possível com mudança de hábitos de vida como adoção de comportamentos saudáveis, adesão aos tratamentos propostos e combate aos fatores de risco citados, como o diabetes mellitus, a hiperlipidemia e a hipertensão arterial (Fernandes, 2009; Smajlovic, 2015; Sirinatnam et al, 2020).

A suspeita de ocorrência do AVE deve ocorrer quando o paciente apresenta um déficit neurológico focal. Portanto, é importante reconhecer os sinais de alerta, que incluem alteração da força e/ou sensibilidade em um ou ambos os lados do corpo, dificuldade visual, dificuldade de fala ou compreensão, vertigem e sensação de desequilíbrio (Chaves et al, 2008).

Para diferenciar os tipos isquêmico e hemorrágico é necessário a realização de exame de imagem (Chaves et al, 2008). De acordo com Cardoso e Meneses (2018), a tomografia computadorizada é uma das principais técnicas de diagnóstico na avaliação inicial do doente com suspeita de AVE e este exame de imagem está indicado para diferenciar AVEi, AVEh de lesões não vasculares. É importante ressaltar também, que o exame de imagem é fundamental para orientar o tratamento, detectar complicações e avaliar o prognóstico do evento neurovascular.

Além das limitações motoras e cognitivas, que são consequências diretas da lesão vascular, há alterações no âmbito emocional, podendo o paciente desenvolver transtornos como os de ansiedade e depressão. Isso afeta diretamente o processo de reabilitação, reduzindo a motivação do doente para se empenhar na recuperação, sendo mais grave quanto mais avançada a idade do paciente (Cancela, 2008).

Em relação às alterações comportamentais, a labilidade emocional é geralmente encontrada. Isso porque os indivíduos apresentam emoções instáveis, sendo capazes de inibir a expressão de emoções espontâneas, o que altera rapidamente o seu comportamento emocional sem haver uma razão aparente e, em muitas vezes, a impossibilidade de exercer atividade remunerada e os gastos com a doença constituem um fenômeno estressor, tendo impactos severos na saúde mental do paciente (Araujo et al, 2011; Cancela, 2008).

Os transtornos psiquiátricos, como os de ansiedade e depressivos tem forte impacto sobre a qualidade de vida do paciente, tendo em vista a percepção que o doente tem quanto ao seu estado de saúde e as sequelas capazes de serem desenvolvidas, segundo estudos de D'aniello et al. (2014) e Gurr e Mullenz (2011). Os interesses e, principalmente, as perspectivas pessoais sofrem mudanças evidentes, rebaixando de forma significativa a qualidade de vida da pessoa acometida 
por AVE. A tendência é que ocorram mudanças bruscas das atividades habituais, limitações antes não experimentadas, e estresse psicológico, principalmente na fase aguda da doença, ou seja, nos três primeiros meses (West, 2010).

Desse modo, a ocorrência de síndromes psiquiátricas no período pós AVE é de etiologia ampla e inclui a limitação física, a piora da qualidade de vida, a presença de sequelas cognitivas, a necessidade de reabilitação e a percepção do próprio paciente em relação ao ambiente. As alterações no âmbito da saúde mental incluem agitação, irritabilidade, falta de iniciativa, apatia, agressividade, desinibição e depressão. Com isso, os pacientes podem vir a se tornar ansiosos e hesitantes, necessitando sempre o apoio de outras pessoas (Fernandes, 2009; Biller et al, 2004).

Dentre as complicações envolvidas, destaca-se a depressão, estando essa condição presente nas fases aguda, subaguda e crônica do evento vascular (Amaral et al, 2020). A prevalência dessa doença varia entre os estudos, no entanto, estima-se que de 10 a 34\% dos pacientes que tiveram AVE terão depressão e conviverão com os sintomas depressivos após a ocorrência do AVE (Fernandes, 2009).

Outros transtornos psiquiátricos envolvidos em quadros pós-AVE são demência vascular, epilepsia, fobia generalizada e transtornos como de pânico, mental orgânico e de ansiedade. Embora menos prevalentes (Fernandes, 2009; Amaral et al, 2020). o diagnóstico destas patologias é fundamental para tratamento adequado destes pacientes.

Considerando a predisposição ao desenvolvimento de doenças psiquiátricas após AVE, este estudo buscou descrever a prevalência destas doenças em pacientes com diagnóstico confirmado de AVE em acompanhamento ambulatorial em um município do oeste paranaense. Além disso, dentro do tema relação entre acidente vascular encefálico e desenvolvimento de síndromes neuropsiquiátricas, serão avaliadas possíveis associações entre sexo e idade superior a 60 anos com desenvolvimento de alguma doença psiquiátrica em específico.

\section{Metodologia}

Trata-se de uma pesquisa retrospectiva, transversal, quantitativa e observacional, com dados advindos do prontuário de dezenove pacientes, com diagnóstico conclusivo de acidente vascular encefálico (AVE) atendidos na clínica e ambulatório da Fundação Hospitalar São Lucas, na cidade de Cascavel-PR, entre os anos de 2015 e 2019, com objetivo de descrever quais doenças psiquiátricas são mais diagnosticadas em pacientes com histórico de AVE, quais medicações são utilizadas e verificar se há associação entre sexo e idade com diagnóstico dos pacientes. Como base metodológica foi utilizado o trabalho de Pereira et al (2018).

A análise estatística foi realizada no software RStudio na versão 1.4.1103. Para as comparações de prevalência entre grupos foram utilizados teste de Fisher e Qui-Quadrado, sendo considerando nível de significância $\alpha=0,05$. Por meio destes testes foram avaliadas as associações entre sexo e idade superior a sessenta anos com doenças psiquiátricas. As tabelas descritivas foram construídas utilizando software Microsoft Excel e Word.

\section{Resultados e Discussão}

Foram analisados prontuários médicos de dezenove pacientes que sofreram AVE e estavam em acompanhamento ambulatorial no serviço de psiquiatria entre os anos de 2015 e 2019. A maioria dos pacientes atendidos apresentava diagnóstico de depressão (53\%), sugerindo que sintomas depressivos após episódio de AVE são muito comuns e frequentes nesta população de pacientes (Amaral et al, 2020). Além disso, a maioria dos pacientes estudados apresenta idade superior a 60 anos (58\%) e utilizava medicação antidepressiva (79\%) (Tabela 1). 
Tabela 1: Informações referentes a pacientes que sofreram AVE e atendidos pelo serviço de psiquiatria da Fundação Hospitalar São Lucas (n=19).

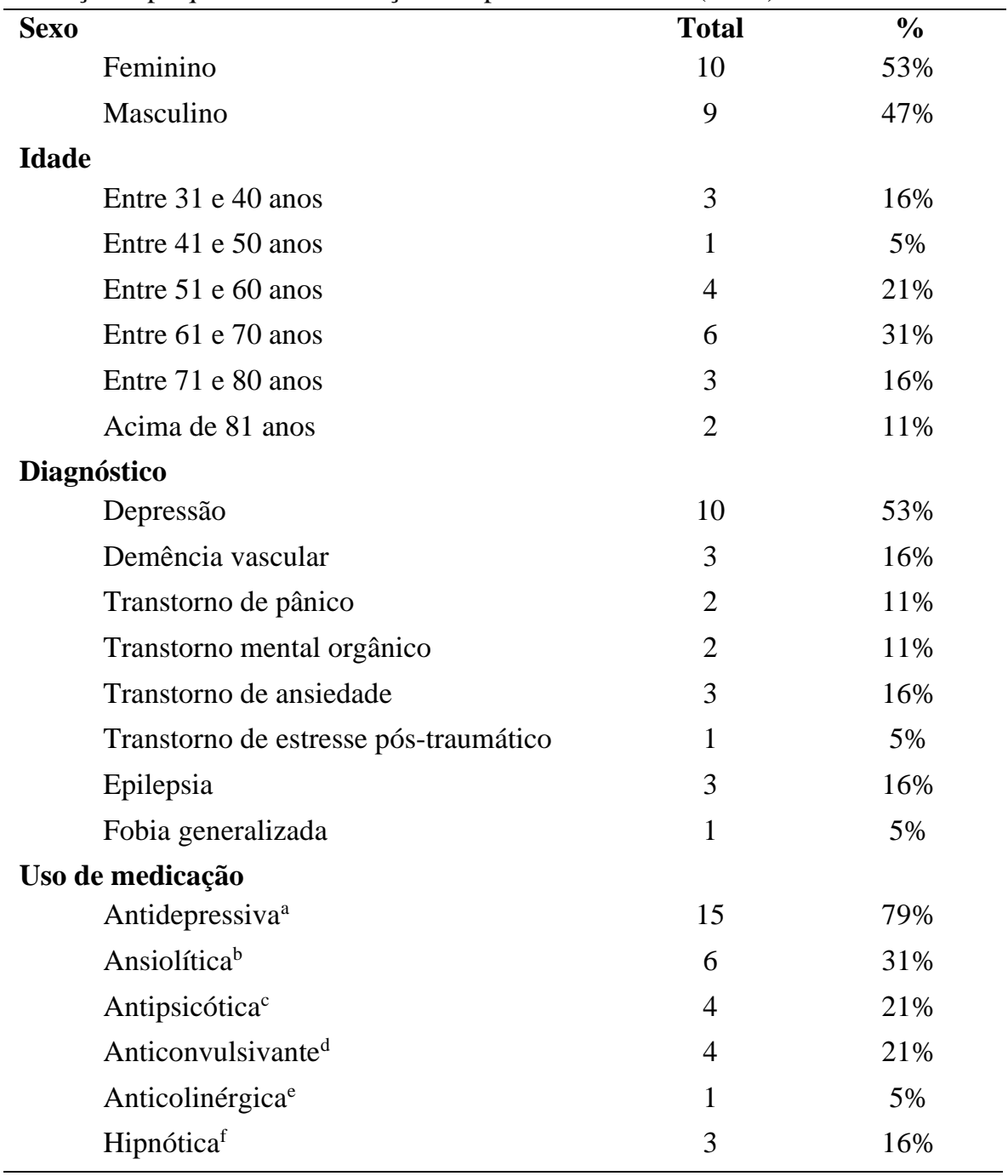

a Foram considerados os medicamentos: Amitriptilina, Duloxetina, Mirtazapina, Venlafaxina, Fluoxetina, Trazodona, Sertralina e Escitalopram

${ }^{\mathrm{b}}$ Foram considerados os medicamentos: Diazepam e clonazepam

${ }^{c}$ Foram considerados os medicamentos: Risperidona, Quetiapina

d Foram considerados os medicamentos: Ácido Valproico, Fenitoína, Fenobarbital e Gabapentina

e Biperideno

${ }^{\mathrm{f}}$ Zolpidem

Fonte: Autores (2021).

Houve associação com significância estatística entre maior prevalência de demência vascular e epilepsia nos pacientes do sexo masculino $(\mathrm{p}=0,05)$. Neste estudo não foi demonstrada a associação entre sexo feminino com maior prevalência de depressão $(\mathrm{p}=0,11)$ nem transtorno de pânico $(\mathrm{p}=0,16)$. Com relação a idade, não houve significância estatística na associação de depressão $(\mathrm{p}=0,84)$, transtorno de ansiedade $(\mathrm{p}=0,74)$ e epilepsia $(\mathrm{p}=0,74)$ em pacientes idosos (Tabela 2). 
Tabela 2: Descrição do valor de p e hipóteses envolvendo sexo e idade de pacientes com histórico de AVE atendidos pelo serviço de psiquiatria da Fundação Hospitalar São Lucas (n=19).

\begin{tabular}{lc}
\hline Sexo Masculino & Valor p \\
\hline Maior prevalência de demência vascular & $0.05^{*}$ \\
Maior prevalência de epilepsia & $0.05^{*}$ \\
Maior prevalência de transtorno mental orgânico & 0.12 \\
Maior prevalência de transtorno de ansiedade & 0.47 \\
\hline Sexo Feminino & Valor p \\
\hline Maior prevalência de depressão & 0.11 \\
Maior prevalência de transtorno de pânico & 0.16 \\
\hline Idade superior a 60 anos & Valor p \\
\hline Depressão & 0.84 \\
Transtorno de ansiedade & 0.74 \\
Epilepsia & 0.74 \\
\hline * denota significância estatística. & \\
Fonte: Pesquisadores (2021). &
\end{tabular}

Foi associado, com significância estatística, a maior prevalência de demência vascular e epilepsia $(\mathrm{p}=0,05)$ nos pacientes do sexo masculino. Como a amostra do estudo foi limitada em dezenove participantes e em seguimento ambulatorial, são necessários novos trabalhos para avaliar se está associação é realmente válida em amostras maiores e que englobam todos os grupos de pacientes. De acordo com dados da literatura a demência não é universal em todos os AVEs, indicando que outros fatores além da isquemia/hemorragia cerebral podem contribuir para ocorrência (ONYIKE, 2006).

Conforme relatado na literatura (Fernandes, 2009; Amaral et al, 2020), a doença psiquiátrica mais diagnosticada em pacientes com histórico de AVE é depressão (53\%). O transtorno de ansiedade também apresentou prevalência considerável (16\%), conforme descrito por Fernandes et al (2009).

Assim como o Perth Community Stroke Study desenvolvido por Burvill et al (1995), o presente estudo não encontrou significância estatística na associação de maior prevalência de depressão no sexo feminino, considerando pacientes com histórico de $\operatorname{AVE}(\mathrm{p}=0,11)$.

\section{Conclusão}

Este estudo, desenvolvido com informações de prontuários ambulatoriais de dezenove pacientes com histórico de AVE (hemorrágico ou isquêmico) cumpriu o objetivo descrever as doenças psiquiátricas mais diagnosticadas nestes pacientes, quais medicações são mais utilizadas e verificar se há associação entre sexo e idade com diagnóstico dos pacientes.

Pode-se concluir deste estudo que patologias psiquiátricas como depressão e ansiedade apresentam elevada prevalência em pacientes com histórico de AVE. Dessa forma, é necessário acompanhamento multiprofissional destes pacientes para diagnosticar e tratar precocemente complicações decorrentes de eventos isquêmicos ou hemorrágicos cerebrais.

A principal limitação do estudo foi a pequena amostra estudada, que reduz o poder do teste (1 - $\beta$ ) nas análises estatísticas realizadas. Futuros trabalhos podem utilizar amostras maiores para confirmar as associações demonstradas por este estudo. Mesmo com limitações, este trabalho pode contribuir com a ciência ao descrever as prevalências de doenças psiquiátricas e a associação de demência vascular e epilepsia com sexo masculino após AVE em um centro de atenção secundário. 


\section{Referências}

Amaral, A. F. S., Ferreira Filho, A. C., Ferreira-Melo, S. E., \& Min, L. L. (2020). Depressão pós acidente vascular cerebral: revisão sistemática.

Araujo, J. S., Silva, S. E. D. da., De Santana, M. E., Conceição, V. M. da, \& Vasconcelos, E. V. (2011). O processo do cuidar/cuidado nas representações sociais de cuidadores de pacientes sequelados por acidente vascular cerebral. Enfermagem Em Foco, 2(4), 235-238. https://doi.org/10.21675/2357-707x.2011.v2.n4.191

Biller, J.; Ruland, S. \& Schneck, M. J. (2004) Ischemic cerebrovascular disease. In: DAROFF, R. B. et al. Neurology in clinical practice (4a ed). Elsevier, 2004.

Burvill, P. W., Johnson, G. A., Jamrozik, K. D., Anderson, C. S., Stewart-Wynne, E. G., \& Chakera, T. M. H. (1995). Prevalence of depression after stroke: The Perth Community Stroke Study. British Journal of Psychiatry, 166(MAR.), 320-327. https://doi.org/10.1192/bjp.166.3.320

Cancela, D. M. G. (2008). O Acidente vascular cerebral - classificação, principais consequências e reabilitação. Portal Dos Psicólogos, 1-18. http://www.psicologia.com.pt/artigos/textos/TL0095.pdf

Caprio, F. Z., \& Sorond, F. A. (2019). Cerebrovascular Disease. Medical Clinics of North America, 103(2), 295-308. https://doi.org/10.1016/j.mcna.2018.10.001

Cardoso A. N. L. \& Meneses R. M. C. (2018) A tomografia computorizada no diagnóstico do AVC agudo. Castelo Branco: Instituto Politécnico de Castelo Branco.

Chaves, M. L. F et al (2008). Rotinas em Neurologia e Neurocirurgia. Artmed.

D’Aniello, G. E., Scarpina, F., Mauro, A., Mori, I., Castelnuovo, G., Bigoni, M., Baudo, S., \& Molinari, E. (2014). Characteristics of anxiety and psychological well-being in chronic post-stroke patients. Journal of the Neurological Sciences, 338(1-2), 191-196. https://doi.org/10.1016/j.jns.2014.01.005

Fernandes, P. T. (2009). Aspectos psicossociais do AVC. ComCiência, 109. http://comciencia.scielo.br/scielo.php?script=sci_arttext\&pid=S1 $51976542009000500027 \& \operatorname{lng}=\mathrm{es} \& n r m=\mathrm{iso} \& \operatorname{lng}=\mathrm{es}$

Gurr, B., \& Muelenz, C. (2011). A follow-up study of psychological problems after stroke. Topics in Stroke Rehabilitation, 18(5), 461-469. https://doi.org/10.1310/tsr1805-461

Hankey, G. J. (2017). Stroke. The Lancet, 389(10069), 641-654. https://doi.org/10.1016/S0140-6736(16)30962-X

Ministério da Saúde. (2019) AVC: o que é, causas, sintomas, tratamentos, diagnóstico e prevenção. www.saude.gov.br/saude-de-a-z/acidente-vascular-cerebralavc.

Onyike, C. U. (2006). Cerebrovascular disease and dementia. International Review of Psychiatry, 18(5), 423-431.

https://doi.org/10.1080/09540260600935421

Pereira A. S. et al. (2018). Metodologia da pesquisa científica. UFSM.

Reis, C., \& Faro, A. (2019). Psychological Repercussions After Stroke: a Literature Review. Psicologia, Saúde \& Doença, 20(1), 16-32. https://doi.org/10.1 530 9/19psd200102

Sarikaya, H., Ferro, J., \& Arnold, M. (2015). Stroke Prevention - Medical and Lifestyle Measures. European Neurology, 73(3-4), 150-157. https://doi.org/10.1159/000367652

Siriratnam, P., Godfrey, A., O’Connor, E., Pearce, D., Hu, C., Low, A., Hair, C., Oqueli, E., Sharma, A., Kraemer, T., \& Sahathevan, R. (2020). Prevalence and risk factors of ischaemic stroke in the young: a regional Australian perspective. Internal Medicine Journal, 50(6), 698-704. https://doi.org/10.1111/imj.14407

Smajlovic, D. (2015). Strokes in young adults: epidemiology and prevention. Vascular Health and Risk Management, 157. https://doi.org/10.2147/VHRM.S53203

Terroni, L. de M. N., Leite, C. C., Tinone, G., \& Fráguas Jr, R. (2003). Depressão pós-AVC: fatores de risco e terapêutica antidepressiva. Revista Da Associação Médica Brasileira, 49(4), 450-459. https://doi.org/10.1590/s0104-42302003000400040

West, R., Hill, K., Hewison, J., Knapp, P., \& House, A. (2010). Psychological disorders after stroke are an important influence on functional outcomes: A prospective cohort study. Stroke, 41(8), 1723-1727. https://doi.org/10.1161/STROKEAHA.110.583351

White, J. H., Magin, P., Attia, J., Sturm, J., Carter, G., \& Pollack, M. (2012). Trajectories of Psychological Distress After Stroke. Annals of Family Medicine, 10(5), 435-442. https://doi.org/10.4324/9781315870052 